\title{
Eutrofização de solos e comunidade arbórea em fragmentos de uma paisagem agrícola
}

\author{
Mariella Camardelli Uzêda(1), Elaine Cristina Cardoso Fidalgo(2), Renato Valadares de Sousa Moreira(1), \\ Ademir Fontana $^{(2)}$ e Guilherme Kangussu Donagemma ${ }^{(2)}$
}

\begin{abstract}
(1)Embrapa Agrobiologia, BR-465, Km 7, Ecologia, CEP 23891-000 Seropédica, RJ, Brasil. E-mail: mariella.uzeda@embrapa.br, renato_vsm@yahoo.com.br (2)Embrapa Solos, Rua Jardim Botânico, no 1.024, Jardim Botânico, CEP 22460-000 Rio de Janeiro, RJ, Brasil. E-mail: elaine.fidalgo@embrapa.br, ademir.fontana@embrapa.br, guilherme.donagemma@embrapa.br
\end{abstract}

Resumo - O objetivo deste trabalho foi avaliar o efeito da eutrofização do solo em fragmentos remanescentes de vegetação nativa sobre a comunidade florística de arbóreas em paisagens agrícolas. Foram amostrados oito sítios de vegetação arbórea na Mata Atlântica, no Estado do Rio de Janeiro, em pares formados de acordo com o uso do solo em seu entorno - intensivo (UI) ou extensivo (UE) - e com diferentes tamanhos: dois fragmentos pequenos, com cerca de 10 ha; dois médios, com 30 ha; dois grandes, com 100 ha; e outros dois sítios localizados dentro de uma área contínua de floresta. Um conjunto de parâmetros bióticos e de solos, além de métricas da paisagem, foi obtido e analisado segundo duas hipóteses: H01, a fertilidade do solo dos remanescentes naturais adjacentes a áreas agrícolas de uso intensivo é alterada; e H02, a eutrofização do solo dos remanescentes naturais adjacentes a áreas agrícolas de uso intensivo altera a composição da sua comunidade de arbóreas. Os teores de cálcio foram cerca de sete vezes maiores nos sítios adjacentes a UI, quando comparados aos fragmentos adjacentes a UE. Os teores de fósforo foram de 1,5 a 2,5 vezes maiores nos fragmentos pequenos e médios adjacentes a áreas de UI. A análise de ordenamento evidenciou que $\mathrm{P}, \mathrm{K}, \mathrm{Ca}$, luz difusa, abertura de dossel, relação área/perímetro e isolamento dos fragmentos explicam $59 \%$ da composição das comunidades arbóreas.

Termos para indexação: deriva, ecologia de paisagens, efeito de borda, fertilidade, serviços ecossistêmicos.

\section{Soil eutrophication and tree community in patches of an agricultural landscape}

\begin{abstract}
This objective of this work was to evaluate the effect of soil eutrophication in remaining patches of native vegetation on the floristic community of trees in agricultural landscapes. Eight sites of arboreal vegetation were sampled in the Atlantic Forest, in state of Rio de Janeiro, Brazil, in pairs formed according to land use in their boundary - intensive (UI) or extensive (UE) -, and with different sizes: two small sites, with about 10 ha; two medium-sized ones, with 30 ha; two large ones, with 100 ha; and two sites located within a continuous forest area. A set of biotic and soil parameters, besides landscape metrics, was surveyed and analyzed considering two hypotheses: H01, soil fertility is changed in forest remnants next to areas of intensive agricultural use; and H02, soil eutrophication in forest remnants next to areas of intensive agricultural use changes the composition of its tree community. Calcium contents were about seven times higher in sites next to UI areas, in comparison with those next to UE areas. Phosphorous contents were 1.5 to 2.5 times higher in the small and medium-sized patches next to UI areas. The ordering analysis showed that $\mathrm{P}, \mathrm{K}, \mathrm{Ca}$, diffuse light, canopy opening, area/perimeter ratio, and patch isolation account for $59 \%$ of the tree community composition.
\end{abstract}

Index terms: spillover, landscape ecology, edge effect, fertility, ecosystem services.

\section{Introdução}

Os solos brasileiros são naturalmente ácidos, com baixa saturação de bases e pequena disponibilidade de fósforo e potássio, em virtude da pobreza em bases do material de origem ou de processos que favorecem a remoção de elementos básicos (Bernardi et al., 2002). Portanto, comunidades bióticas vinculadas a esses solos se adaptaram e evoluíram em condições de baixa disponibilidade de nutrientes. A entrada de nutrientes exógenos em locais de baixa fertilidade pode alterar a composição botânica natural e aumentar a taxa de mortalidade de algumas espécies de plantas nativas, ou privilegiar o desenvolvimento e abundância de outras (Hobbs \& Huenneke, 1992; Thomson \& Leishman, 2004).

A fragmentação de ambientes apresenta como principal consequência os efeitos de borda (Laurance et al., 2007). Esses efeitos atuam em resposta à interação entre o ambiente natural e o antropizado, e 
pode alterar padrões e processos bióticos e abióticos (Bierregaard Jr. et al., 1992). O grau de severidade desses efeitos está diretamente ligado à intensidade do manejo adotado, que depende da quantidade de insumos aplicados e das práticas de mobilização do solo nas áreas adjacentes ao fragmento, que atuam sobre os processos que ocorrem a partir da borda dos remanescentes de vegetação nativa (Chabrerie et al., 2012).

O tamanho e o isolamento na paisagem são aspectos importantes, que interagem com os efeitos de borda e influenciam a sobrevivência das espécies em paisagens fragmentadas (Martensen et al., 2008). De maneira geral, fragmentos maiores possuem maior abundância e diversidade de recursos naturais, o que lhes permite abrigar maior número de espécies e maiores populações, e aumenta sua capacidade de responder a variações ambientais e de manter o processo de regeneração.

A Mata Atlântica, hoje, é um bioma fortemente antropizado, ainda que abrigue um número importante de espécies endêmicas, as quais fazem do bioma ser relevante em biodiversidade para as iniciativas de conservação (Morellato \& Haddad, 2000). Como cerca de $80 \%$ dos fragmentos são menores que 50 ha, e tem alto grau de isolamento (Ribeiro et al., 2009), o planejamento do uso das terras requer abordagens mais integradoras, que considerem as demandas produtivas e de conservação.

Ainda que a configuração da paisagem tenha um papel importante sobre processos ecossistêmicos, a intensidade de uso da matriz tem se mostrado um importante vetor de conservação da diversidade, destacadamente em paisagens agrícolas, onde ambientes seminaturais, não raras vezes, passam a abrigar espécies de maior plasticidade (Ries et al., 2004).

A intensificação de cultivo, fundamentada na maior adoção de insumos químicos e de maquinário, tem sido considerada uma estratégia viável para a redução da pressão sobre áreas ainda conservadas ou destinadas à conservação. O sistema chamado de poupa-terra, do inglês "land sparing", considera que o aumento da produtividade reduziria a pressão da agricultura sobre os remanescentes de áreas naturais, uma vez que minimizaria a conversão dos ecossistemas naturais em áreas produtivas (Tscharntke et al., 2012). Entretanto, Didham et al. (2015) alertam que ignorar o reflexo do manejo adotado nos diferentes usos do solo sobre a escala da paisagem é um dos equívocos ocasionados por abordagens compartimentalizadas.

Apesar da demanda latente por práticas produtivas que colaborem com a conservação, este trabalho é o primeiro a abordar os reflexos das práticas de cultivo sobre a fertilidade do solo em remanescentes florestais de Mata Atlântica, bem como suas consequências sobre a comunidade arbórea. As hipóteses foram testadas de forma a possibilitar a identificação de práticas de cultivo ou estratégias de manejo da paisagem que permitam auxiliar a conservação da biodiversidade em paisagens agrícolas, em que: H01 testou se a fertilidade do solo dos remanescentes naturais adjacentes a áreas agrícolas de uso intensivo é alterada; e H02, se a eutrofização do solo dos remanescentes naturais adjacentes a áreas agrícolas de uso intensivo provoca a alteração da composição da sua comunidade de arbóreas.

O objetivo deste trabalho foi avaliar o efeito da eutrofização do solo em fragmentos remanescentes de vegetação nativa sobre a comunidade florística de arbóreas em paisagens agrícolas.

\section{Material e Métodos}

A área de estudo foi a bacia hidrográfica do rio Guapi-Macacu, localizada na parte leste da baía de Guanabara, no Estado do Rio de Janeiro. Os três municípios nos quais a bacia está inserida (Cachoeiras de Macacu, Guapimirim e Itaboraí) somam um território de $1.740 \mathrm{~km}^{2}$, correspondendo a quase $4 \%$ do território e 2\% do Estado.

$\mathrm{Na}$ área, predomina clima do tipo tropical, com verão úmido e chuvoso e inverno frio, com pouca chuva. A temperatura média anual é de $21^{\circ} \mathrm{C}$, e a precipitação média anual varia de $1.700 \mathrm{~mm}$ a $2.600 \mathrm{~mm}$ (Comperj, 2011).

As terras da bacia são ocupadas predominantemente por florestas em diversos estádios de sucessão (45\%) e por pastagens $(43,6 \%)$ (Fidalgo et al., 2008). A agricultura tem destaque nas áreas de baixada, principalmente do Município de Cachoeiras de Macacu, RJ, e tem como principais culturas o aipim, o milho e o inhame.

Para seleção dos fragmentos, foram adotados os critérios de estrutura da vegetação nativa (dada pela estratificação, porte das árvores e formas de vida), manejo e histórico de uso.

Neste estudo, o uso intensivo (UI) foi representado por áreas ocupadas por cultivo de milho-verde (Zea 
mays) rotacionado com o cultivo de mandioca (Manihot esculenta Crantz).Trata-se, portanto, de um sistema produtivo dependente do frequente revolvimento do solo, para o plantio das culturas, e uso de insumos agroquímicos. O cultivo do milho se dá, nesse sistema, por dois ciclos da cultura, de 90 dias, entre novembro e junho. A inserção da cultura da mandioca ocorre a partir do mês de maio.

O preparo do solo caracteriza-se pela aração seguida de gradagem. Para o cultivo do milho são adicionadas, em média, duas toneladas de calcário por hectare e $60 \mathrm{~kg} \mathrm{ha}^{-1}$ de adubo do tipo 4/14/8. Alguns dos proprietários utilizam composto orgânico, aplicado em cobertura, como complemento à adubação química. No geral, agroquímicos são utilizados no controle de pragas e doenças. Para o cultivo da mandioca, é realizado novo preparo do solo (aração seguida de gradagem). Para essa cultura, é aproveitado o efeito residual da adubação aplicada para a cultura do milho.

As áreas com pastagem, formadas com Urochloa brizantha, representaram o sistema extensivo (UE), no qual ocorre pastoreio rotacionado com a alternância periódica do rebanho para outras pastagens da propriedade. Os pastos são regularmente limpos, com uso de roçagem e queima, e a lotação é, em média, de 1 cabeça por ha.

Para avaliação do efeito do uso do solo, foram selecionadas áreas de vegetação nativa, cujo entorno de área antrópica (agroecossistema) em contato direto com a borda do fragmento tivesse uma extensão mínima de 100x100 m. Os sítios de amostragem foram estabelecidos na face das áreas voltadas para a área produtiva. Ao todo, foram selecionadas oito áreas, sendo seis fragmentos e duas áreas dentro do Parque Estadual dos Três Picos (áreas contínuas). Das áreas selecionadas, quatro possuem entorno de UI e quatro de UE, formando pares quanto ao tamanho: pequenos (cerca de $10 \mathrm{ha}$ ), médios (cerca de $20 \mathrm{ha}$ ), grandes (entre 100 e 200 ha) e as áreas contínuas situadas no interior do Parque Estadual dos Três Picos (49.259,25 $\mathrm{km}^{2}$ ). Em todos os fragmentos selecionados, o agroecossistema vizinho possui entre 10 e 15 anos de uso contínuo seguindo uma mesma rotina de manejo. A localização dos sítios estudados na bacia do rio GuapiMacacu está destacada na Figura 1.

Foram obtidos inicialmente os seguintes parâmetros bióticos: área basal, densidade relativa, dominância relativa e percentual dos diferentes estágios sucessionais: pioneiras $(\mathrm{Pi})$, secundárias iniciais $(\mathrm{Si})$ e secundárias tardias $(\mathrm{St})$. Como métricas da paisagem, foram obtidas por fragmento: área, razão área/ perímetro, raio de giro, índice de dimensão fractal, círculo circunscrito, índice de contiguidade, índice de proximidade e distância euclidiana do vizinho mais próximo. A seleção dos parâmetros bióticos e das métricas da paisagem selecionadas para este trabalho está fundamentada em Uzêda et al. (2011), visando obter uma representatividade da paisagem e evitar o uso de parâmetros e métricas altamente correlacionadas.

Os levantamentos florísticos tiveram início em maio de 2007, tendo sido concluídos em novembro de 2011. O levantamento pedológico e amostragem de solos, assim como as fotografias de dossel, foram realizados em maio de 2011.

Os sítios selecionados foram divididos em ambientes, borda, porção interior e área com predomínio de clareiras, que se caracterizam da seguinte forma: borda, ambiente mais próximo ao sistema produtivo, que sofre forte influência antrópica em virtude da forte exposição ao vento, à luz e a outros efeitos decorrentes do uso; porção intermediária; parte situada no interior do fragmento, posterior à borda, geralmente mais preservada, ainda que, a depender do tamanho do fragmento, seu formato e manejo, sofra impacto dos efeitos de borda; e área com predomínio de clareiras, parte do fragmento onde interferências naturais provocaram queda de árvores, e, de maneira geral, apresentam maior abertura de dossel.

Cabe salientar que a identificação desses ambientes, visando a uma maior representatividade de todo o fragmento, permitiu identificar os ambientes em que determinadas variáveis se destacavam. Mas não é o principal objetivo deste trabalho estabelecer uma comparação entre esses ambientes, e sim entre os sítios como um todo.

Foram estabelecidas nove parcelas de $50 \times 5 \mathrm{~m}$, em cada um dos sítios; três em cada um dos ambientes descritos acima. Essas parcelas foram estabelecidas com $10 \mathrm{~m}$ de distância na horizontal e vertical entre elas, tendo-se formado um gradiente diagonal que representava uma média dos ambientes avaliados. A primeira parcela do ambiente de borda foi alocada a $20 \mathrm{~m}$ do final do cultivo, para dentro do fragmento, tendo-se definido $20 \mathrm{~m}$ como distância entre a última parcela de um ambiente e a primeira parcela do ambiente seguinte. A avaliação florística da comunidade arbórea foi realizada, conforme descrito 
abaixo, na área das parcelas acima mencionadas. As parcelas centrais foram tomadas como referência de localização para a abertura de um pequeno perfil na forma de minitrincheira $(0,8 \times 0,5 \times 0,8 \mathrm{~m})$, para avaliação morfológica, caracterização química e física, e para classificação dos solos com indicação da classe predominante em cada um dos ambientes dos fragmentos avaliados.

Para avaliação da fertilidade do solo, foram coletadas amostras nas profundidades de 0 a $0,10 \mathrm{~m} \mathrm{e}$ de 0,10 a $0,20 \mathrm{~m}$. Entretanto, em análises prévias dos resultados obtidos, não foram identificadas diferenças entre a profundidade entre 0,10 e $0,20 \mathrm{~m}$ e aquela entre 0 e $0,10 \mathrm{~m}$. Sendo assim, optou-se por utilizar apenas os resultados obtidos na primeira camada.

Em cada uma das três parcelas implantadas, foram retiradas três amostras simples de solo, no total de nove amostras para cada um dos ambientes avaliados (borda, porção intermediária e área com predomínio de clareiras); portanto, 18 amostras por sítio, nas duas profundidades amostrais $(0-0,10$ e $0,10-0,20 \mathrm{~m})$. Esse mesmo padrão de amostragem foi utilizado para a obtenção das fotografias de dossel e das medidas de abertura (Ados) e luz difusa (Ldif). Em cada uma das parcelas, foram retiradas três fotografias, posicionadas nas extremidades e no centro, tendo-se formado um conjunto de nove repetições por ambiente.

Para avaliação florística da comunidade de arbóreas, em cada uma das parcelas, os indivíduos adultos com circunferência à altura do peito (CAP) maiores ou iguais a $15 \mathrm{~cm}$, foram medidos e identificados. Com base na medida da circunferência, foram calculadas as variáveis de estrutura fitossociológica, de acordo com MuellerDombois \& Ellenberg (1974). Neste trabalho, calculouse o índice de valor de importância (IVI) para cada uma das espécies encontradas (Curtis \& McIntosh, 1951).

Para obtenção do valor de Ados e de Ldif, foram obtidas fotografias hemisféricas com uma máquina fotográfica Nikon, modelo Coolpix 4500, acoplada a uma lente olho-de-peixe Nikon $8 \mathrm{~mm}$ (Nikon FC-
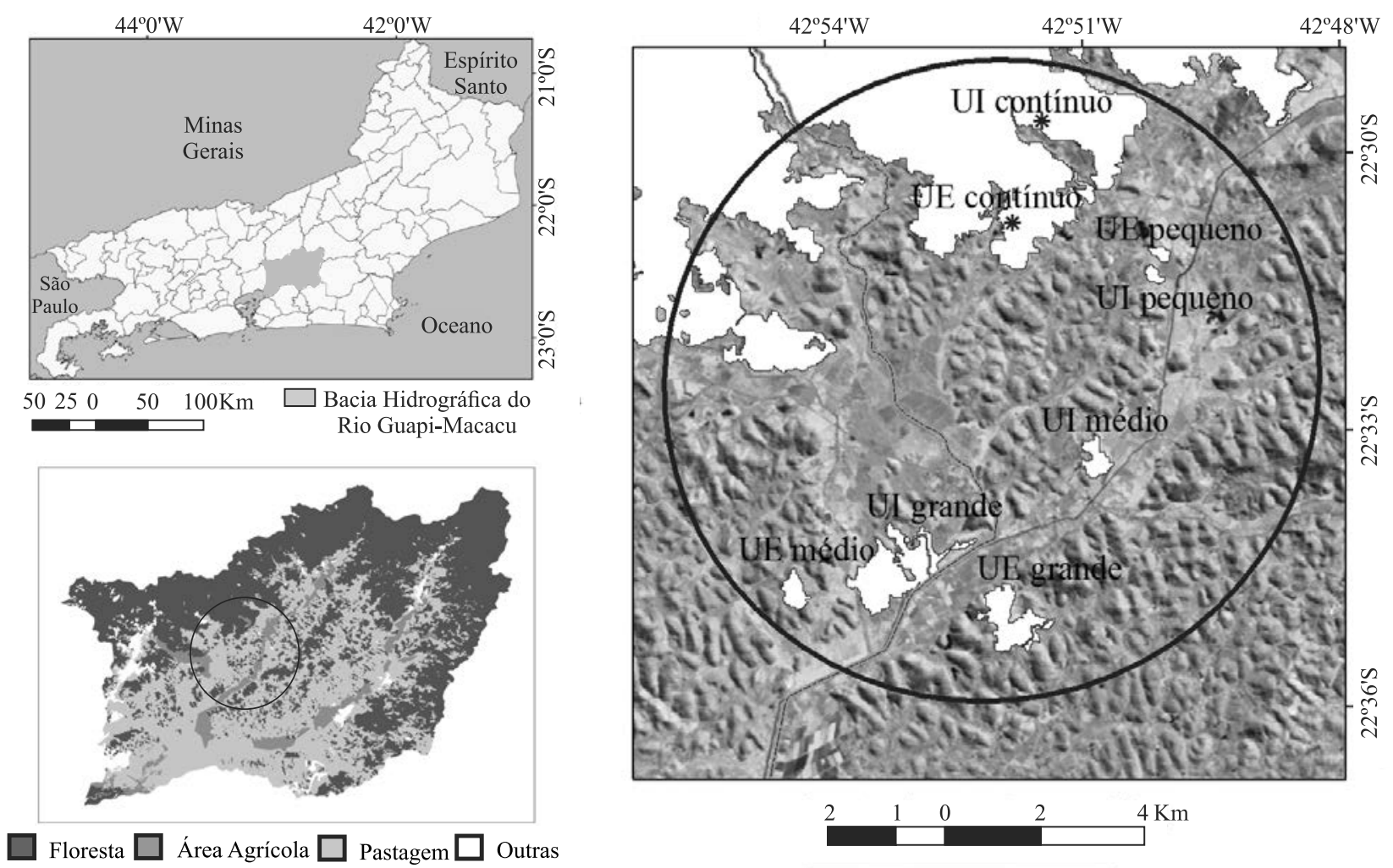

Figura 1. Localização geográfica dos sítios amostrados na bacia Guapi-Macacu, Rio de Janeiro, RJ. 
08). As imagens foram posteriormente binarizadas no programa Sidelook 1.0 (Nobis \& Hunziker, 2005), e analisadas com auxílio do programa Gap Light Analyser Version 2.0 (Frazer et al., 1999).

Para avaliação das características morfológicas do solo, como cor, estrutura, textura e sequência de horizontes, seguiram-se as recomendações do Manual de Descrição e Coleta de Solo no Campo (Santos et al., 2013b). Os atributos físicos (granulometria) e químicos ( $\mathrm{pH}$ em água, cálcio, $\mathrm{Ca}^{2+}$, magnésio, $\mathrm{Mg}^{2+}$, potássio, $\mathrm{K}^{+}$e $\mathrm{P}$ disponível foram analisados conforme recomendação da Embrapa (Donagema et al., 2011), e os solos foram classificados de acordo com critérios estabelecidos pelo Sistema Brasileiro de Classificação de Solos (Santos et al., 2013a).

O mapa de uso e cobertura da terra das bacias hidrográficas dos rios Guapi-Macacu e Caceribu em 2007, em escala 1:50.000 (Pedreira et al., 2009), foi utilizado para a identificação das áreas sob cobertura de vegetação natural da área de estudo. Esse mapa foi elaborado com base na classificação de imagens do sensor TM-Landsat 5, de junho a agosto de 2007, com resolução de 30 metros.

O mapa original foi recortado para o limite da área da bacia do Guapi-Macacu, e extraídos os sítios de vegetação natural selecionados: floresta em estágios inicial, médio e avançado de regeneração. Essas áreas, para efeito de simplificação, são denominadas neste trabalho como fragmentos ou remanescentes de vegetação natural.

Os dados de fragmentos foram espacializados, em formato digital, "raster", com resolução de 30 metros, e foi utilizado o programa Fragstats (Mcgarigal, 2011) para cálculo das métricas da paisagem. Foram utilizadas as medidas de: área (AREA), que se refere ao tamanho do fragmento; razão área/perímetro (PARA), que é um indicador do formato do fragmento e, portanto, relacionado à quantidade de borda existente; e distância euclidiana do vizinho mais próximo (ENN), que é um indicador de isolamento do fragmento.

Para obtenção da vizinhança com o uso agrícola (limagr), foram observados seus limites com outros usos, delimitados no mesmo mapa de uso e cobertura utilizado. Calculou-se qual a proporção de seus limites que faz divisa com os diferentes tipos de uso da terra. Pelo fato que os fragmentos estudados são circundados apenas por pastagem e agricultura e, portanto, os percentuais desses dois tipos de limites totalizam 100, optou-se por utilizar apenas o percentual de limite com agricultura e, com isso, eliminar o restante da informação, uma vez que elas seriam altamente correlacionadas.

Os métodos de análise foram selecionados visando à obtenção de um padrão que explique os fenômenos avaliados, evitando análise particularizada dos sítios. Portanto, para todas as análises realizadas, os parâmetros bióticos e abióticos se referem à média do conjunto de informações encontradas, considerando o conjunto de parcelas e ambientes de cada um dos oito sítios selecionados.

Para análise da primeira hipótese, foram construídos e testados modelos lineares generalizados, considerando as seguintes variáveis independentes como determinantes dos níveis de fertilidade do solo: tipo de solo limite com área agrícola, abertura de dossel, tamanho do remanescente e relação área/ perímetro. $\mathrm{O}$ modelo que contrapõe a primeira hipótese proposta (modelo nulo) inclui apenas o tipo de solo, considerando que os níveis de fertilidade dos solos encontrados se devem exclusivamente ao material de origem e, portanto, não há contaminação. Dessa forma, ainda que os solos dos sítios avaliados sejam diferentes, é possível avaliar se há ou não contaminação do solo pela deriva de adubos e corretivos.

Considerando a distribuição Gaussiana dos dados, foram testados 16 modelos ao todo, e a comparação e seleção do modelo mais adequado foi feita com os valores da segunda ordem de critérios Akaike (AICc) (Burnham \& Anderson, 2002). Foram selecionados os modelos com valores de $\triangle \mathrm{AICc}$ menores que dois e de maior peso (AICcWi). A avaliação do ajuste dos parâmetros dos modelos selecionados foi realizada por meio de um teste de Qui-quadrado. As análises dos modelos envolveram os pacotes "bbmle", versão 1.0.16, e "MuMIn", versão 1.15.1, ambos atrelados ao programa estatístico $R$, versão 3.2.2 (R Core Team, 2008).

$\mathrm{Na}$ análise da segunda hipótese, para explicar dissimilaridades entre as comunidades arbóreas dos fragmentos, foi utilizada a análise de escalonamento multidimensional não métrico (NMDS), com o índice de similaridade de Bray-Curtis. (Dufrêne \& Legendre, 1997), fundamentado no índice de valor de importância (IVI). Para essa análise, foram utilizados os pacotes “vegan", versão 2.3.0, e o "labdsv", versão 1.7.0, também associados ao programa estatístico R.

A contribuição dos parâmetros abióticos e das variáveis estruturais da paisagem para a dissimilaridade encontrada foi avaliada por meio de 
análise multivariada não paramétrica da variância NPManova, ("Non-parametric multivariate analysis of variance"), usando a distância euclidiana (Anderson, 2001). A significância do teste foi avaliada utilizando teste F com base em 999 permutações sequenciais da soma dos quadrados dos dados transformados para o intervalo entre 0 e 1 (range). Cada uma das variáveis foi comparada de forma pareada entre os sítios de mesma escala de tamanho. Os métodos aqui utilizados têm sido exercitados em trabalhos recentes (Duncan et al., 2008; Chabrerie et al., 2012; Didham et al., 2015), atendendo com acurácia à análise dos dados obtidos.

\section{Resultados e Discussão}

Os solos encontrados nos fragmentos avaliados indicaram variações naturais, influenciadas pelo relevo. A classe dos Cambissolos Háplicos dominou principalmente a borda dos fragmentos. Nos ambientes de área com predomínio de clareiras, predominou a classe dos Latossolos Vermelho-Amarelos. Nas áreas de porção intermediária, foram encontradas as classes Latossolos Amarelos, Argissolos Amarelos e Argissolos Vermelho-Amarelos (Tabela 1).

Ao avaliar a fertilidade do solo nos fragmentos estudados, foram encontradas evidências de eutrofização do solo, ainda que as alterações encontradas não tenham reflexo claro sobre o $\mathrm{pH}$. Os teores de cálcio e magnésio foram maiores nos sítios adjacentes a áreas de UI, destacadamente nas áreas de borda e de predominância de clareiras. Para o cálcio, esse padrão de resultados foi mais nítido, tendo-se encontrado valores de $\mathrm{Ca}$ cerca de 7,0 vezes maiores nos ambientes dos sítios adjacentes a UI (Tabela 1).

A relação entre a intensidade do manejo nos sistemas produtivos e seus reflexos sobre a fertilidade do solo nos fragmentos adjacentes ainda é pouco explorada na literatura. Entretanto, Chabrerie et al. (2012), ao avaliar o impacto da intensidade do cultivo no entorno de fragmentos florestais, constataram haver substituição das espécies locais por espécies calcífilas e nitrófilas na borda de áreas avaliadas, em resposta a sistemas de cultivos de maior intensidade. Isso é representativo do processo de eutrofização do solo em virtude da entrada de cálcio oriundo das áreas de cultivo.

O constante movimento de preparo do solo, seguido da calagem, práticas comuns nas áreas de cultivo da região, potencializam a entrada de cálcio nos fragmentos adjacentes a essas áreas.
Os resultados obtidos para o potássio apresentaram variações apenas nos ambientes de borda, onde os teores foram 1,5 vezes maiores nos sítios adjacentes a UI, quando comparados aos valores encontrados para os mesmos ambientes adjacentes a UE. Trabalhos recentes concluem que, independentemente do tamanho dos fragmentos, as áreas de borda são mais vulneráveis ao uso do solo adjacente a elas e apresentam alterações de grande importância na fertilidade do solo (Duncan et al., 2008; Chabrerie et al., 2012; Didham et al. 2015). Dessa forma, a adoção de barreiras físicas (Didham et al., 2015) e de práticas agrícolas que permitam o menor movimento do solo, a exemplo do plantio direto, pode ser importante ferramenta na conservação da biodiversidade em paisagens agrícolas.

O fósforo, por sua vez, apresentou valores de 1,5 a 2,5 vezes maiores, em todos os ambientes dos fragmentos pequenos e médios adjacentes a áreas de UI, quando comparados aos sítios de mesmo tamanho adjacentes a áreas de UE. A pouca mobilidade do fósforo no solo possibilita o acúmulo desse nutriente oriundo de sucessivos eventos de contaminação, recentes e pretéritos, e evidencia uma tendência à eutrofização dos solos dos fragmentos menores vizinhos a áreas de UI.

Entretanto, os expressivos valores de $\mathrm{P}$ e $\mathrm{K}$ obtidos nos sítios pertencentes ao fragmento grande adjacente a UE, e nas áreas contínuas adjacentes a UE e UI, indicam que o efeito da deriva pode ter maior alcance, ao colocar também grandes áreas de vegetação natural, que não possuem áreas de uso intensivo no seu entorno, em situação vulnerável (Tabela 1). O vento e seus padrões de ocorrência causam a contaminação do solo mesmo em grandes remanescentes, ainda que os fragmentos pequenos sigam apresentando maior vulnerabilidade à deriva dos nutrientes e agroquímicos utilizados nas áreas agrícolas (Duncan et al., 2008).

Os modelos avaliados para explicar o padrão observado de fertilidade do solo utilizaram os parâmetros abióticos avaliados (Tabela 1) e os resultados encontrados para as métricas da paisagem (Tabela 2). O modelo selecionado para $\mathrm{Ca}$ e $\mathrm{Mg}$ indicou a existência de interação significativa entre os tipos de solo (Solo) e o limite de área agrícola (limagr) $[y=\beta 0+\beta 1$ Solo $+\beta 2$ limagr $+\beta 3$ (Solo limagr); $\mathrm{X}^{2}=0,01$ e $\mathrm{X}^{2}=0,01$, para $\mathrm{Ca}$ e $\mathrm{Mg}$, respectivamente]. Quando apenas o tipo de solo foi considerado, ainda que a variável tenha tido contribuição significativa (y $=\beta 0+\beta 1$ Solo; Solo, $\mathrm{X}^{2}=0,07$ e $\mathrm{X}^{2}=0,05$, para Ca e

Pesq. agropec. bras., Brasília, v.51, n.9, p.1120-1130, set. 2016 DOI: 10.1590/S0100-204X2016000900011 
$\mathrm{Mg}$, respectivamente), o modelo ganhou altos valores de $\mathrm{AICc}(22,0$ e 20,0, para Ca e Mg, respectivamente), o que o torna menos representativo que o modelo selecionado e confirma a primeira hipótese proposta (Tabela 3). Assim, os teores de $\mathrm{Ca}$ e $\mathrm{Mg}$ encontrados se deveram não só ao material de origem, mas também ao uso intenso do solo nas áreas adjacentes.

No modelo explicativo para os teores de potássio, a interação limagrAREA $[y=\beta 0+\beta 1$ limagr $+\beta 2$ AREA
+ $\beta 3$ (limagr AREA); limagrAREA: $\left.X^{2}=0,03144\right]$ destacou-se. Quanto ao fósforo, o modelo que melhor explicou os níveis de $\mathrm{P}$ evidencia forte interação entre a abertura de dossel e o limite de área agrícola, assim como entre o tamanho das áreas de vegetação nativa e sua relação perímetro/área $[\mathrm{y}=\beta 0+\beta 1 \operatorname{Ados}+\beta 2$ limagr $+\beta 3$ (Ados limagr) $+\beta 4$ AREA $+\beta 5$ PARA $+\beta 6$ (AREAPARA); Adoslimagr: $X^{2}=0,05$; AREAPARA: $\left.\mathrm{X}^{2}=0,0013\right]$. Para os elementos $\mathrm{P}$ e $\mathrm{K}$, o tipo de

Tabela 1. Parâmetros abióticos avaliados em áreas de remanescentes florestais, na bacia Guapi-Macacu, Rio de Janeiro, $\mathrm{RJ}^{(1)}$.

\begin{tabular}{|c|c|c|c|c|c|c|c|c|}
\hline \multirow{2}{*}{$\begin{array}{l}\text { Uso/tamanho } \\
\text { das áreas }\end{array}$} & \multicolumn{8}{|c|}{ Parâmetros abióticos } \\
\hline & Classificação & $\mathrm{pH}$ & $\mathrm{P}\left(\mathrm{mg} \mathrm{kg}^{-1}\right)$ & $\mathrm{Ca}\left(\mathrm{mmol}_{\mathrm{c}} \mathrm{dm}^{-3}\right)$ & $\mathrm{Mg}\left(\mathrm{mmol}_{\mathrm{c}} \mathrm{dm}^{-3}\right)$ & $\mathrm{K}\left(\mathrm{mg} \mathrm{kg}^{-1}\right)$ & Ados & Ldif \\
\hline & \multicolumn{8}{|c|}{ Borda } \\
\hline UI pequeno & $\begin{array}{l}\text { Latossolo Vermelho- } \\
\text { Amarelo }\end{array}$ & $3,84(0,24)$ & $4,96(0,22)$ & $13,7(0,16)$ & $5,4(0,6)$ & $52,10(11,58)$ & $12,07(1,71)$ & $2,29(0,28)$ \\
\hline UE pequeno & Cambissolo Háplico & $3,58(0,43)$ & $1,97(0,46)$ & $2,0(02,3)$ & $0,4(0,6)$ & $34,00(6,85)$ & $15,12(1,89)$ & $2,83(0,26)$ \\
\hline UI médio & Cambissolo Háplico & $3,95(0,40)$ & $2,05(0,36)$ & $4,3(3,2)$ & $2,7(00,9)$ & $60,78(23,76)$ & $12,17(2,21)$ & $1,90(0,34)$ \\
\hline UE médio & Latossolo Amarelo & $3,30(0,34)$ & $0,92(0,53)$ & $0,6(1,3)$ & $1,8(01,2)$ & $35,11(6,81)$ & $16,32(4,47)$ & $3,34(0,98)$ \\
\hline UI grande & Cambissolo Háplico & $4,02(0,19)$ & $1,90(0,23)$ & $1,0(2,8)$ & $1,4(01,0)$ & $50,67(15,08)$ & $12,13(1,74)$ & $2,45(0,33)$ \\
\hline UE grande & Argissolo Amarelo & $3,83(0,12)$ & $4,97(1,67)$ & $0,8(2,3)$ & $0,8(02,2)$ & $53,89(8,63)$ & $14,28(2,23)$ & $2,96(1,59)$ \\
\hline UI contínuo & Cambissolo Háplico & $4,16(0,13)$ & $4,23(1,56)$ & $3,9(4,4)$ & $1,7(01,9)$ & $81,33(15,91)$ & $12,32(1,74)$ & $5,57(0,77)$ \\
\hline UE contínuo & Latossolo Amarelo & $3,84(0,07)$ & $3,41(0,62)$ & $0,0(0,0)$ & $0,0(00,0)$ & $45,78(9,70)$ & $10,58(1,26)$ & $4,67(1,02)$ \\
\hline & \multicolumn{8}{|c|}{ Porção interior } \\
\hline UI pequeno & $\begin{array}{l}\text { Argissolo Vermelho- } \\
\text { Amarelo }\end{array}$ & $3,46(0,07)$ & $4,91(1,10)$ & $0,0(0,0)$ & $0,4(0,2)$ & $40,67(5,29)$ & $12,18(1,00)$ & $2,56(0,17)$ \\
\hline UE pequeno & $\begin{array}{l}\text { Latossolo Vermelho- } \\
\text { Amarelo }\end{array}$ & $3,76(0,94)$ & $2,16(0,28)$ & $1,3(1,8)$ & $0,2(0,2)$ & $43,00(6,96)$ & $14,60(3,33)$ & $3,12(0,68)$ \\
\hline UI médio & $\begin{array}{l}\text { Latossolo Vermelho- } \\
\text { Amarelo }\end{array}$ & $3,71(0,16)$ & $2,55(0,42)$ & $8,6(3,7)$ & $1,6(1,6)$ & $39,50(12,28)$ & $10,04(2,11)$ & $1,93(0,36)$ \\
\hline UE médio & $\begin{array}{l}\text { Latossolo Vermelho- } \\
\text { Amarelo }\end{array}$ & $3,12(0,24)$ & $1,62(0,72)$ & $3,5(2,6)$ & $1,4(0,9)$ & $36,00(5,08)$ & $12,07(1,63)$ & $2,58(0,39)$ \\
\hline UI grande & $\begin{array}{l}\text { Latossolo Vermelho- } \\
\text { Amarelo }\end{array}$ & $3,29(0,27)$ & $1,48(0,19)$ & $0,0(0,0)$ & $1,6(0,7)$ & $40,14(4,12)$ & $11,05(1,79)$ & $2,37(0,37)$ \\
\hline UE grande & Argissolo Amarelo & $3,29(0,27)$ & $5,09(1,35)$ & $0,0(0,0)$ & $0,0(0,0)$ & $55,56(9,51)$ & $12,34(4,07)$ & $2,33(0,59)$ \\
\hline UI contínuo & $\begin{array}{l}\text { Latossolo Vermelho- } \\
\text { Amarelo }\end{array}$ & $3,94(0,08)$ & $2,28(1,04)$ & $3,9(4,4)$ & $1,7(1,9)$ & $68,33(11,37)$ & $10,55(1,11)$ & $4,62(0,39)$ \\
\hline \multirow[t]{2}{*}{ UE contínuo } & Latossolo Amarelo & $4,04(0,03)$ & $3,78(0,87)$ & $0,0(0,0)$ & $0,0(0,0)$ & $48,67(8,16)$ & $9,29(1,45)$ & $3,99(0,77)$ \\
\hline & \multicolumn{8}{|c|}{ Predomínio de clareiras } \\
\hline UI pequeno & Cambissolo Háplico & $3,52(0,14)$ & $6,37(1,08)$ & $9,7(0,8)$ & $4,6(0,4)$ & $42,44(8,42)$ & $11,80(1,08)$ & $2,36(0,23)$ \\
\hline UE pequeno & Latossolo Amarelo & $3,25(0,42)$ & $2,40(0,48)$ & $1,3(1,5)$ & $0,7(0,6)$ & $33,86(7,19)$ & $13,69(3,02)$ & $2,80(0,73)$ \\
\hline UI médio & Latossolo Amarelo & $3,71(0,16)$ & $2,76(0,48)$ & $0,0(0,0)$ & $0,4(0,4)$ & $26,94(4,41)$ & $11,09(1,92)$ & $1,84(0,27)$ \\
\hline UE médio & $\begin{array}{l}\text { Latossolo Vermelho- } \\
\text { Amarelo }\end{array}$ & $3,05(0,16)$ & $1,02(0,39)$ & $0,3(0,5)$ & $2,3(1,1)$ & $23,11(5,59)$ & $12,33(2,25)$ & $2,46(0,42)$ \\
\hline UI grande & $\begin{array}{l}\text { Latossolo Vermelho- } \\
\text { Amarelo }\end{array}$ & $3,61(0,08)$ & $2,12(0,35)$ & $0,0(0,0)$ & $0,3(0,2)$ & $35,67(5,44)$ & $11,89(1,54)$ & $2,47(0,30)$ \\
\hline UE grande & $\begin{array}{l}\text { Latossolo Vermelho- } \\
\text { Amarelo }\end{array}$ & $3,79(0,06)$ & $3,93(0,47)$ & $0,0(0,0)$ & $0,0(0,0)$ & $45,50(4,65)$ & $7,37(3,24)$ & $1,51(0,56)$ \\
\hline UI contínuo & Argissolo Amarelo & $3,85(0,20)$ & $3,06(1,18)$ & $3,8(4,3)$ & $1,9(2,2)$ & $48,89(15,77)$ & $10,93(1,92)$ & $4,70(1,22)$ \\
\hline UE contínuo & $\begin{array}{l}\text { Latossolo Vermelho- } \\
\text { Amarelo }\end{array}$ & $4,00(0,08)$ & $6,18(5,44)$ & $1,0(2,8)$ & $0,5(1,3)$ & $42,22(10,85)$ & $10,37(1,41)$ & $4,35(0,88)$ \\
\hline
\end{tabular}

${ }^{(1)}$ Os valores representam a média das nove amostras obtidas em cada um dos ambientes, e entre parênteses o seu respectivo desvio-padrão. UI, uso intensivo; UE, uso extensivo; Ados, abertura de dossel; e Ldif, luz difusa. 
Tabela 2. Resultados das métricas da paisagem obtidos para os sítios amostrados na bacia Guapi-Macacu, Rio de Janeiro, RJ.

\begin{tabular}{|c|c|c|c|c|}
\hline Uso/tamanho da área & Limagr & AREA & PARA & ENN \\
\hline Uso extensivo/ pequeno & 0,0 & 8,5 & 184,4 & 192,1 \\
\hline Uso extensivo/ médio & 0,0 & 26,5 & 113,4 & 60,0 \\
\hline Uso extensivo/grande & 9,0 & 168,1 & 113,8 & 150,0 \\
\hline Uso extensivo/ contínua ${ }^{(1)}$ & 0,0 & $49.259,3$ & 30,4 & 60,0 \\
\hline Uso intensivo/ pequeno & 67,3 & 8,8 & 176,9 & 60,0 \\
\hline Uso intensivo/ médio & 15,6 & 29,3 & 124,7 & 161,6 \\
\hline Uso intensivo/grande & 8,8 & 90,4 & 124,2 & 90,0 \\
\hline Uso intensivo/ contínua ${ }^{(2)}$ & 24,5 & $49.259,3$ & 30,4 & 60,0 \\
\hline
\end{tabular}

(1)Área natural contínua, adjacente a agroecossistema de uso extensivo (UE). ${ }^{(2)}$ Área natural contínua, adjacente a agroecossistema de uso intensivo (UI). Limagr, limite da área agrícola; AREA, tamanho das áreas de remanescentes avaliadas; PARA, relação área/perímetro; e ENN, distância euclidiana para o fragmento mais próximo.

Tabela 3. Síntese dos modelos avaliados e selecionados para cada variável dependente ${ }^{(1)}$, mostrando os modelos selecionados $^{(2)}$ e o modelo nulo.

\begin{tabular}{|c|c|c|c|c|}
\hline Modelos avaliados & Modelo selecionado e modelo nulo & $\Delta \mathrm{AICc}$ & AICcWi & $\mathrm{X}^{2(2,3)}$ \\
\hline $\mathrm{y}=\beta 0+\beta 1$ Solo $; y=\beta 0+\beta 1$ limagr; $y=\beta 0+\beta 1$ Solo $+\beta 2$ limagr & $\mathrm{Ca}$ & & & \\
\hline$y=\beta 0+\beta 1$ limagr $+\beta 2$ AREA $; y=\beta 0+\beta 1$ Solo $+\beta 2$ limagr $+\beta 3$ (Solo & $y=\beta 0+\beta 1$ Solo $+\beta 2$ limagr $+\beta 3$ ( Solo limagr $)$ & 0,0 & 0,8552 & $0,01003 *$ \\
\hline limagr); $y=\beta 0+\beta 1$ limagr $+\beta 2$ AREA $+\beta 3$ (limagr AREA); $y=\beta 0+$ & $\mathrm{Y}=\beta 0+\beta 1$ Solo & 22,0 & $<0,001$ & 0,06998 \\
\hline$\beta 1$ Solo $+\beta 2$ Ados $+\beta 2$ limagr + B4PARA $; y=\beta 0+\beta 1$ Solo $+\beta 2$ Ados $^{-}$ & $\mathrm{Mg}$ & & & \\
\hline $\begin{array}{l}+\beta 3 \text { limagr }+\beta 4 \text { AREA } ; y=\beta 0+\beta 1 \text { Solo }+\beta 2 \text { limagr }+\beta 3 \text { AREA }+ \\
\beta 4 \text { PARA } y=\beta 0+\beta 1 \text { Ado }+\beta 2 \text { limagr }+\beta 3 \text { PARA }+\beta 4 \text { AREA } \cdot y\end{array}$ & $y=\beta 0+\beta 1$ Solo $+\beta 2$ limagr $+\beta 3$ ( Solo limagr $)$ & 0,0 & 0,7343 & $0,01241 *$ \\
\hline$=\beta 0+\beta 1$ Ados $+\beta 2$ limagr $+\beta 3($ Ados limagr $)+\beta 4$ AREA; $y=\beta 0$ & $\mathrm{Y}=\beta 0+\beta 1 \underline{\text { Solo }}$ & 20,0 & $<0,001$ & 0,05474 \\
\hline$+\beta 1$ Solo $+\beta 2$ Ados $+\beta 3$ limagr + B4PARA $+\beta 5$ AREA $; y=\beta 0+$ & $\mathrm{P}$ & & & \\
\hline $\begin{array}{l}\beta 1 \text { Ados }+\beta 2 \text { limagr }+\beta 3 \text { (Ados limagr) }+\beta 4 \text { AREA }+\beta 5 \text { Solo } ; y=\beta 0 \\
+\beta 1 \text { Ados }+\beta 2 \text { limagr }+\beta 3 \text { (Ados limagr }+\beta 4 \text { AREA }+\beta 5 \text { PARA } ;+\end{array}$ & $\begin{aligned} y= & \beta 0+\beta 1 \text { Ados }+\beta 2 \text { limagr }+\beta 3(\text { Ados limagr })+ \\
& \beta 4 \text { AREA }+\beta 5 \text { PARA }+\beta 6(\underline{\text { AREA PARA })}\end{aligned}$ & 0,0 & 0,5040 & $\begin{array}{l}0,046332 * \mathrm{e} \\
0,001291 * *\end{array}$ \\
\hline$\beta 6($ AREA PARA $) ; y=\beta 0+\beta 1$ Ados $+\beta 2$ PARA $+\beta 3($ Ados PARA $)+$ & $\mathrm{Y}=\beta 0+\beta 1 \underline{\text { Solo }}$ & 14,0 & $<0,001$ & 0,2547 \\
\hline$\beta 4$ limagr $+\beta 5$ AREA $+\beta 6($ limagr AREA $) ; y=\beta 0+\beta 1$ Ados + & $\mathrm{K}$ & & & \\
\hline$\beta 2$ AREA $+\beta 3($ Ados AREA $)+\beta 4$ PARA $+\beta 5$ limagr $+\beta 6$ (PARA & $=\beta 0+\beta 1$ limagr $+\beta 2 \mathrm{AREA}+\beta 3($ limagr AREA $)$ & 0,0 & 0,4079 & $0,03144^{*}$ \\
\hline limagr) & $\mathrm{Y}=\beta 0+\beta 1 \underline{\text { Solo }}$ & 8,3 & 0,0064 & 0,4788 \\
\hline
\end{tabular}

${ }^{(1)} \mathrm{Ca}$, cálcio; $\mathrm{Mg}$, magnésio; $\mathrm{P}$, fósforo; e K, potássio. As variáveis independentes foram tipo de solo (Solo), limite da área agrícola (limagr), tamanho dos fragmentos (AREA), relação perímetro/área (PARA) e abertura de dossel (Ados). ${ }^{(2)}\left(\triangle \mathrm{AICc}<2\right.$ e X ${ }^{2}$ ). ${ }^{*}{ }^{* *}$ Significativo a 5 e $1 \%$ de probabilidade, respectivamente. ${ }^{(3)}$ Referem-se a variável independente sublinhada do modelo selecionado ou nulo.

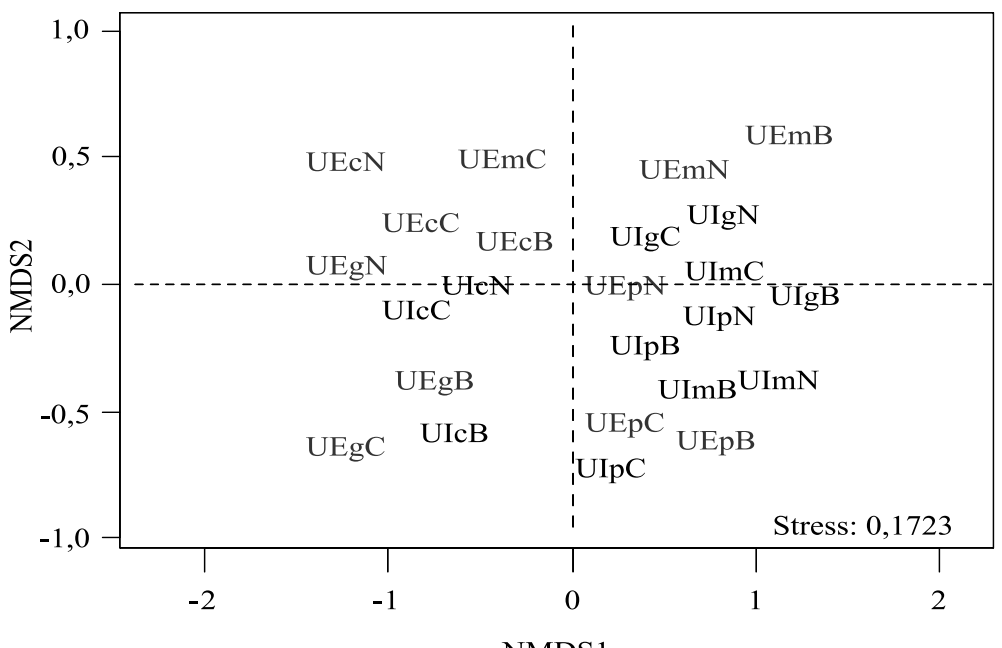

NMDS1

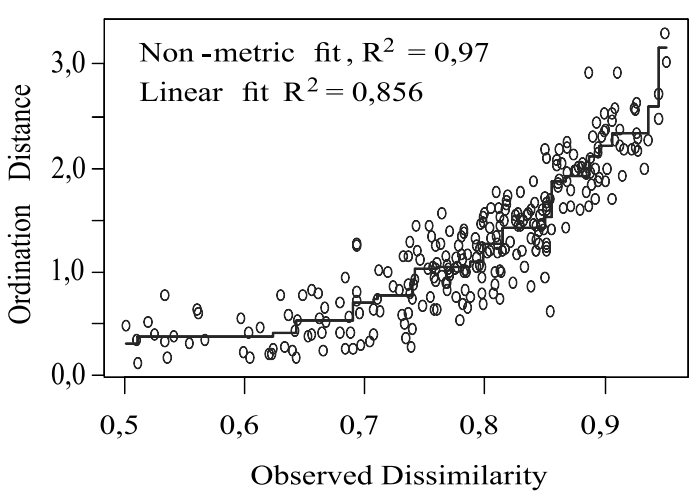

Observed Dissimilarity

Figura 2. Escalonamento multidimensional não métrico da composição da comunidade de arbóreas avaliadas em ambientes de borda $(\mathrm{B})$, porção intermediária $(\mathrm{N})$ e em área com predomínio de clareiras $(\mathrm{C})$, nos fragmentos pequeno $(\mathrm{p})$, médio $(\mathrm{m})$ e grande (g) e em áreas naturais contínuas (c), adjacentes a agroecossistemas de uso intensivo (UI) e de uso extensivo (UE), na bacia do rio Guapi-Macacu, Rio de Janeiro, RJ. 
solo não exerceu influência nos níveis de fertilidade observados, o que levou à retirada da variável "Solos" do modelo; a contribuição dessa variável no modelo nulo não foi significativa $\left(y=\beta 0+\beta 1\right.$ Solo; $X^{2}=0,25$ e $\mathrm{X}^{2}=0,48$, para $\mathrm{P}$ e $\mathrm{K}$, respectivamente) (Tabela 3 ). Duncan et al. (2008), ao trabalhar em áreas de solos pobres voltadas a produção de grãos, no Semiárido australiano, relataram que a interação entre tamanho e localização do fragmento quanto à direção dos ventos predominantes é o elemento determinante do modelo que melhor explica os resultados para $\mathrm{P}$ e $\mathrm{K}$.

$\mathrm{O}$ escalonamento multidimensional da composição da comunidade arbórea (Figura 2), fundamentada no índice de valor de importância (IVI) das espécies, refletiu as diferenças de composição entre as comunidades de arbóreas dos sítios (stress de 0,17 e $\left.\mathrm{R}^{2}=0,97\right)$. Os sítios pertencentes a fragmentos adjacentes a áreas de UE encontram-se concentrados, predominantemente, no quadrante esquerdo do gráfico, juntamente com as áreas contínuas adjacentes a agroecossistemas de UI.

A análise multivariada (NPManova) revelou que $59 \%$ da variação da composição da comunidade de arbóreas, entre os diferentes sítios avaliados, foi explicada pelos parâmetros abióticos e pelas métricas da paisagem. Tiveram contribuição significativa os seguintes parâmetros: fósforo $(\mathrm{P}, 7 \% ; \mathrm{p}=0,03)$, luz

Tabela 4. Análise multivariada não paramétrica da variância (NPManova) para a contribuição dos parâmetros abióticos na dissimilaridade das comunidades de arbóreas avaliadas na bacia do rio Guapi-Macacu, Rio de Janeiro, RJ.

\begin{tabular}{lrccccc}
\hline Parâmetro $^{(1)}$ & Gl & SQ & QM & F & $\mathrm{R}^{2}$ & $\mathrm{p}$ \\
\hline Solo & 1 & 0,31 & 0,31 & 1,39 & 0,05 & 0,107 \\
$\mathrm{Ca}$ & 1 & 0,32 & 0,32 & 1,44 & 0,05 & 0,071 \\
$\mathrm{Mg}$ & 1 & 0,29 & 0,29 & 1,29 & 0,04 & 0,139 \\
$\mathrm{P}$ & 1 & 0,48 & 0,48 & 2,15 & 0,07 & $0,003^{* *}$ \\
$\mathrm{~K}$ & 1 & 0,39 & 0,39 & 1,79 & 0,06 & $0,027^{*}$ \\
Ados & 1 & 0,40 & 0,40 & 1,80 & 0,06 & $0,024^{*}$ \\
Ldif & 1 & 0,44 & 0,44 & 2,01 & 0,07 & $0,010^{* *}$ \\
limagr & 1 & 0,34 & 0,34 & 1,56 & 0,05 & $0,045^{*}$ \\
AREA & 1 & 0,23 & 0,23 & 1,04 & 0,04 & 0,361 \\
PARA & 1 & 0,37 & 0,37 & 1,68 & 0,06 & $0,040^{*}$ \\
ENN & 1 & 0,32 & 0,32 & 1,44 & 0,05 & 0,088 \\
\hline Resíduo & 12 & 2,65 & 0,22 & - & 0,41 & - \\
\hline Total & 23 & 6,5439 & - & - & 1,0 & - \\
\hline
\end{tabular}

${ }^{(1)}$ Solo, tipo de solo; Ados, abertura de dossel; Ldif, luz difusa; limagr, limite da área agrícola; AREA, tamanho dos fragmentos; PARA, relação perímetro/área, e ENN, distância euclidiana para o fragmento mais próximo. *e**Significativo a 5 e $1 \%$ de probabilidade, respectivamente. difusa (Ldif, 7\%; $\mathrm{p}=0,01$ ), abertura de dossel (Ados, $6 \% ; \mathrm{p}=0,024)$, potássio $(\mathrm{K}, 6 \% ; \mathrm{p}=0,027)$, cálcio $(\mathrm{Ca}$, $5 \% ; \mathrm{p}=0,071$ ), relação área/perímetro (PARA, 6\%; $\mathrm{p}=0,04)$ e isolamento $(\mathrm{ENN}, 5 \% ; \mathrm{p}=0,088)$ (Tabela 4). O tipo de solo (Solo), os teores de magnésio ( $\mathrm{Mg}$ ) e o tamanho das áreas avaliadas (AREA) não tiveram participação significativa na composição das comunidades arbóreas dos diferentes sítios. Portanto, o menor isolamento, a menor exposição ao ambiente externo e a manutenção da estrutura do dossel das áreas de vegetação nativa foram fundamentais para conservação da comunidade de arbóreas. Dessa forma, além das barreiras físicas no entorno dos fragmentos, ações de enriquecimento terão grande contribuição à redução do processo de contaminação.

Estudos semelhantes em outros biomas e em outras condições de paisagem são importantes para reunir informações que permitam indicar sistemas de cultivo que contribuam na conservação dos fragmentos florestais.

\section{Conclusões}

1. Fragmentos florestais adjacentes a áreas de uso intensivo são vulneráveis à contaminação e à consequente eutrofização do solo e, nas condições deste estudo, a eutrofização deve-se, principalmente, à entrada de cálcio e fósforo.

2. Bordas, áreas com maior abertura de dossel - como os ambientes onde predominam clareiras - e fragmentos de menor porte (até 30 ha) estão mais expostos à eutrofização.

3. A contaminação por fósforo, potássio e cálcio pode provocar alterações na comunidade de arbóreas.

4. A estrutura do dossel e a configuração da paisagem influenciam a definição das espécies de arbóreas nos fragmentos; em que maiores relações perímetro/área do fragmento - ou seja, a maior exposição ao ambiente externo - e o maior isolamento do fragmento o tornam mais suscetível à perda de espécies.

\section{Agradecimentos}

Aos proprietários das áreas pela autorização para realização do trabalho; ao Fundo Nacional do Meio Ambiente e à Embrapa, pelo apoio financeiro e logístico para realização do trabalho. 


\section{Referências}

ANDERSON, M.J. A new method for non-parametric multivariate analysis of variance. Austral Ecology, v.26, p.32-46, 2001. DOI: 10.1111/j.1442-9993.2001.01070.pp.x.

BERNARDI, A.C.C.; MACHADO, P.L.O. de A.; SILVA, C.A. Fertilidade do solo e demanda por nutrientes no Brasil. In: MANZATTO, C.V.; FREITAS JÚNIOR, E. de; PERES, J.R.R. (Ed.). Uso agrícola dos solos brasileiros. Rio de janeiro: Embrapa Solos, 2002. p.61-77.

BIERREGAARD JR., R.O.; LOVEJOY, T.E.; KAPOS, V.; SANTOS, A.A. dos; HUTCHINGS, R.W. The biological dynamics of tropical rainforest fragments. BioScience, v.42, p.859-866, 1992. DOI: $10.2307 / 1312085$.

BURNHAM, K.P.; ANDERSON, D.R. Model selection and multimodel inference. $2^{\text {nd }}$ ed. New York: Springer, 2002. 488p.

CHABRERIE, O.; JAMONEAU, A.; GALLET-MOURON, E.; DECOCQ, G. Maturation of forest edges is constrained by neighbouring agricultural land management. Journal of Vegetation Science, v.24, p.58-69, 2012. DOI: 10.1111/j.16541103.2012.01449.x.

COMPERJ. Agenda 21: cachoeiras de Macacu. 2011. Disponível em: <http://www.agenda21 comperj.com.br/sites/localhost/files/ Cachoeiras.pdf $>$. Acesso em: 18 out. 2013.

CURTIS, J.T.; MCINTOSH, R.P. The interrelations of certain analytic and synthetic phytosociological characters. Ecology, v.31, p.476-496, 1951. DOI: 10.2307/1931725.

DIDHAM, R.K.; BARKER, G.M.; BARTLAM, S.; DEAKIN, E.L.; DENMEAD, L.H.; FISK, L.M.; PETERS, J.M.R.; TYLIANAKIS, J.M.; WRIGHT, H.R.; SCHIPPER, L.A. Agricultural intensification exacerbates spillover effects on soil biogeochemistry in adjacent forest remnants. PLoS ONE, v.10, p.1-32, 2015. DOI: 10.1371/journal.pone.0116474.

DUFRÊNE, M.; LEGENDRE, P. Species assemblages and indicator species: the need for a flexible asymmetrical approach. Ecological Monographs, v.67, p.345-366, 1997. DOI: 10.1890/0012-9615(1997)067[0345:SAAIST]2.0.CO;2.

DUNCAN, D.H.; DORROUGH, J.; WHITE, M.; MOXHAM, C. Blowing in the wind? Nutrient enrichment of remnant woodlands in an agricultural landscape. Landscape Ecology, v.23, p.107-119, 2008. DOI: 10.1007/s10980-007-9160-0.

DONAGEMA, G.; CAMPOS, D.; CALDERANO, S.; TEIXEIRA, W.; VIANA, J. Manual de métodos de análise de solo. 2.ed. rev. Rio de Janeiro: Embrapa Solos, 2011. 230p. (Embrapa Solos. Documentos, 132).

FIDALGO, E.C.C.; PEDREIRA, B. da C.C.G.; ABREU, M.B. de; MOURA, I.B. de; GODOY, M.D.P. Uso e cobertura da terra na bacia hidrográfica do rio Guapi-Macacu. Rio de Janeiro: Embrapa Solos, 2008. 31p. (Embrapa Solos. Documentos, 105).

FRAZER, G.W.; CANHAM, C.D.; LERTZMAN, K.P. Gap Light Analyzer (GLA): imaging software to extract canopy structure and gap light transmission indices from true-colour fisheye photographs: user's manual and program documentation, version
2.0. Burnaby: Simon Fraser University; Millbrook: Institute of Ecosystem Studies, 1999. 36p.

HOBBS, R.J.; HUENNEKE, L.F. Disturbance, diversity and invasion: implications for conservation. Conservation Biology, v.6, p.324-337, 1992. DOI: 10.1046/j.15231739.1992.06030324.x.

LAURANCE, W.F.; NASCIMENTO, H.E.M.; LAURANCE, S.G.; ANDRADE, A.; EWERS, R.M.; HARMS, K.E.; LUIZÃO, R.C.C.; RIBEIRO, J.E. Habitat fragmentation, variable edge effects, and the landscape-divergence hypothesis. PLoS ONE, v.2, p.1-8, 2007. DOI: 10.1371/journal.pone.0001017.

MARTENSEN, A.C.; PIMENTEL, R.G.; METZGER, J.P. Relative effects of fragment size and connectivity on bird community in the Atlantic Rain Forest: implications for conservation. Biological Conservation, v.141, p.2184-2192, 2008. DOI: 10.1016/j. biocon.2008.06.008.

MCGARIGAL, K. Fragstats: user guideline. Version 3. Disponível em: <http://www.umass.edu/landeco/research/ fragstats/documents/User\%20guidelines/User\%20guidelines $\% 20$ content.htm>. Acesso em: 9 set. 2011.

MORELLATO, L.P.C.; HADDAD, C.F.B. Introduction: the Brazilian Atlantic Forest. Biotropica, v.32, p.786-792, 2000. DOI: 10.1111/j.1744-7429.2000.tb00618.x.

MUELlER-DOMBOIS, D.; ELlENBERG, D. Aims and methods of vegetation ecology. New York: J. Wiley, 1974. 547p.

NOBIS, M.; HUNZIKER, U. Automatic thresholding for hemispherical canopy-photographs based on edge detection. Agricultural and Forest Meteorology, v.128, p.243-250, 2005. DOI: 10.1016/j.agrformet.2004.10.002.

PEDREIRA, B. da C.C.G.; FIDALGO, E.C.C; PRADO, R.B.; FADUL, M.J. do A.; BASTOS, E.C.; SILVA, S.A. da; ZAINER, N.G.; PELUZO, J. Dinâmica de uso e cobertura da terra nas bacias hidrográficas do Guapi-Macacu e Caceribu - RJ. Rio de Janeiro: Embrapa Solos, 2009. 66p. (Embrapa Solos. Boletim de pesquisa e desenvolvimento, 136).

R CORE TEAM. R: the $\mathrm{R}$ project for statistical computing. Vienna: R Foundation for Statistical Computing, 2011. Version 3.2.2. Disponível em: $<$ https://www.r-project.org/>. Acesso em: 7 ago. 2015.

RIBEIRO, M.C.; METZGER, J.P.; MARTENSEN, A.C.; PONZONI, F.; HIROTA, M.M. The Brazilian Atlantic forest: how much is left and how is the remaining forest distributed? Implications for conservation. Biological Conservation, v.142, p.1141-1153, 2009. DOI: 10.1016/j.biocon.2009.02.021.

RIES, L.; FLETCHER JR, R.J.; BATTIN, J.; SISK, T.D. Ecological responses to habitat edges: mechanisms, models, and variability explained. Annual Review of Ecology, Evolution and Systematics, v.35, p.491-522, 2004. DOI: 10.1146/annurev. ecolsys.35.112202.130148.

SANTOS, H.G. dos; JACOMINE, P.K.T.; ANJOS, L.H.C. dos; OLIVEIRA, V.A. de; LUMBRERAS, J.F.; COELHO, M.R.; ALMEIDA, J.A. de; CUNHA, T.J.F.; OLIVEIRA, J.B. de. Sistema brasileiro de classificação de solos. 3.ed. rev. e ampl. Brasília: Embrapa. 2013a. 353p. 
SANTOS, R.D. dos; LEMOS, R.C. de; SANTOS, H.G. dos; KER, J.C.; ANJOS, L.H.C. dos; SHIMIZU, S.H. Manual de descrição e coleta de solo no campo. 6.ed. rev. e ampl. Viçosa: Sociedade Brasileira de Ciência do Solo, 2013b. 100p.

THOMSON, V.P.; LEISHMAN, M.R. Survival of native plants of Hawkesbury Sandstone communities with additional nutrients: effect of plant age and habitat. Australiam Journal of Botany, v.52, p.141-147, 2004. DOI: 10.1071/BT03047.

TSCHARNTKE, T.; CLOUGH, Y.; WANGER, T.C.; JACKSON,L.; MOTZKE. I.: PERFECTO. I.: VANDERMEER. J.: WHITBREAD.
A. Global food security, biodiversity conservation and the future of agricultural intensification. Biological Conservation, v.151, p.5359, 2012. DOI: 10.1016/j.biocon.2012.01.068.

UZÊDA, M.C.; FIDALGO, E.C.C.; IGUATEMY, M. de A.; ALVES, R.C.; ROUWS, J.R.C. Explorando as relações entre estrutura da paisagem e atributos de qualidade de fragmentos em região de Mata Atlântica no estado do Rio de Janeiro. Rio de Janeiro: Embrapa Solos, 2011. 31p. (Embrapa Solos. Boletim de pesquisa e desenvolvimento, 190).

Recebido em 31 de agosto de 2015 e aprovado em 3 de fevereiro de 2016 